\title{
Viewpoint: Reply to Comment
}

\section{on 'Using NMR to Test Molecular Mobility during a \\ Chemical Reaction'}

Tian Huang ${ }^{a}$ Huan Wang, ${ }^{b}$ and Steve Granick ${ }^{a, c, *}$

${ }^{\text {a }}$ Center for Soft and Living Matter, Institute for Basic Science (IBS), Ulsan 44919, South Korea

${ }^{\mathrm{b}}$ College of Chemistry and Molecular Engineering, Peking University, Beijing, 100871, P. R. China

${ }^{\mathrm{c}}$ Departments of Chemistry and Physics, Ulsan National Institute of Science and Technology

(UNIST), Ulsan 44919, South Korea

* Corresponding author email: sgranick@gmail.com

ABSTRACT: Reports of boosted diffusion during chemical and enzymatic reactions have inspired a loyal community of scientists who find them so counter-intuitive that they must be artifact. This second Comment on the subject by these authors is about technicalities of how to analyze data we deposited online regarding J. Phys. Chem. Lett. (2021) 12, 2370 and Science (2020) 369, 537. Now that their own data is also online, one apparent discrepancy can be resolved: we demonstrate that the authors' data agrees with ours because their first Comment on this subject reported only truncated short-time excerpts of the longer time series they deposited online (zenodo.org/record/4628353). This second Comment adds 5 additional objections, 4 of which are too technical to change the qualitative conclusion. The $5^{\text {th }}$ objection errs because it omits to 
recognize intermediate states of the click reaction during which one reactant complexes with the catalyst to form an object of larger size. Elsewhere we analyzed in great detail the respective influences of boosted diffusion and this hydrodynamic effect (doi.org/10.26434/chemrxiv.14740563.v1). The factual evidence and reasoning in this Reply strongly support this laboratory's earlier conclusions regarding boosted diffusion during common chemical reactions. 
To some researchers, reports in the literature about boosted mobility during certain chemical and enzymatic reactions ${ }^{1-9}$ are so counter-intuitive that they feel their colleagues must have measured artifact. This is the context of the present Viewpoint ${ }^{10}$ expressing concerns about technicalities of how to analyze data we deposited online ${ }^{11-12}$ regarding the click reaction ${ }^{1-2}$. Beyond using NMR data, we also presented evidence for boosted diffusion based on microfluidics measurements ${ }^{1}$ and additional chemical reactions than the click reaction, ${ }^{1}$ but this Comment does not address those measurements. Although we disagree with the authors, we sympathize because our original measurements in this field were in fact motivated by our own original skepticism. We changed our minds based on the evidence.

In an initial round of Comment-Response, these authors asserted that they were unable to reproduce our data ${ }^{13}$, we responded that we could not reproduce their assertions ${ }^{14}$, and after publication the authors, complying with journal policy, deposited their raw data online ${ }^{15}$. We have analyzed it with two salient conclusions. First, these authors published only the data at the start of reaction, $<20 \%$ of reaction ("Germany" dataset) and $<35 \%$ reaction ("Australia" dataset). Quality of the NMR data in the "Germany" dataset is poor -- we use this word advisedly and encourage readers to consult the raw data, illustrated in Fig. 1, to see for themselves. But the "Australia" data is excellent quality. Fig. 1B shows the "Australia" dataset does not depend on magnetic field sequence (random, increasing or decreasing). This is consistent with data from this laboratory ${ }^{2}$. But inconsistent with the authors' claims ${ }^{13}$.

We do not understand why assertions in the authors' first Comment on this subject ${ }^{13}$ are not supported by their own data. Reasonable researchers can disagree how to select the limits of data integration or about how to separate NMR peaks, but that secondary issue can affect 
significant figures only. Regarding whether the data are broadly consistent qualitatively - or not - the data agree. We invite readers to look at Fig. 1 and judge for themselves.

Inquiring minds might wonder why the inter-laboratory comparison in Fig. 1 does not include the azide reactant. The reason is that the authors of this Comment refused us permission to publish data they shared with us privately at an early stage of this disagreement.

Our Reply to the authors' first Comment ${ }^{14}$ was followed by our longer paper amplifying $\mathrm{it}^{2}$. In this, their second Comment, the authors now raise objections to our long paper. Technical objections (i) and (iv) of this Comment repeat the assertion that measurements depend on the order of magnetic field gradient imposed, but as just discussed, they are contradicted by the authors' data and ours (Fig. 1).

The authors here present 5 new critiques:

First: The authors would have been happier if we had analyzed absolute value of diffusion coefficient. Our original publication, prepared over 1 year ago, had normalized data to its value after reaction ended. Our thought at the time was to facilitate comparison between laboratories by eliminating issues of instrument calibration. Now, the authors correctly show that the absolute value of the alkyne reagent decreases with time, but their critique does not appreciate that the alkyne reagent participates in intermediate states of the click reaction. These transient complexes with catalyst are geometrically larger than the original reactant. Elsewhere we analyzed in detail the respective influences of boosted diffusion and this hydrodynamic effect ${ }^{16}$. Our analysis in that paper superseded that in earlier papers from this laboratory in which consequences of complexation with the catalyst were not considered, but the qualitative conclusion of boosted diffusion was unaffected ${ }^{16}$. 
In Fig. 2 we remind readers of the click reactions' catalytic cycle, in which transient coordination of the alkyne reagent to form complexes with catalyst is evident. Elsewhere we demonstrate boosted diffusion of the relevant reaction intermediate ${ }^{16}$.

More direct is to analyze diffusion of a reagent whose intermediate-state complexes are too short-lived to be captured by these NMR measurements. Representative data are shown in Fig. 3 regarding the azide reagent (click reaction) and norbornene reagent (Grubbs reaction). These data for two independent chemical systems agree qualitatively in the sense that they begin at one number, rise during the chemical reaction, and finally return to their original value (the experimental design to make this plain involves using mismatched reactant stoichiometry $\left.{ }^{16}\right)$. To generate these data points from the raw NMR spectra we used automated methods of NMR peak integration, described elsewhere ${ }^{16}$ to improve on the manual integration we had used in the original publications ${ }^{1-2}$. These data illustrate why we insist on the conclusion of boosted diffusion.

Second: The Comment asserts that overlapping NMR peaks led to incorrect data analysis. Reasonable people can usually disagree about the details of technical analysis, so we choose to respond by asking whether issues raised by this Comment could have changed the conclusions qualitatively. An easy way to approach this is to analyze peak height (obvious to the eye) instead of integrated area. Plotting reagent diffusion coefficient against reaction time, Fig. 4 shows agreement between the peak height and integrated area for both reagents of the click reaction. We could quibble about numbers but the qualitative agreement is evident.

Third: The authors of the Comment are unhappy that intensity of the NMR water signal changed with time. We remind readers that the NMR signal intensity depends on proton exchange rate with surrounding species in this complex hydrogen bonded network. It is an 
interesting question whether the overall spin-lattice relaxation time $T_{1}$ of water increases, during the click reaction, beyond the $2-4 \mathrm{~s}$ that one finds in the literature ${ }^{17}$, and we plan to investigate this in the future. We varied delay times in our earlier paper ${ }^{1}, 3$ to $10 \mathrm{~s}$, and obtained consistent findings ${ }^{2}$. One should notice that $\mathrm{T}_{1}$ is also related to the molecular tumbling rate such that $\mathrm{T}_{1}$ increases when molecular motion slows ${ }^{18}$.

The Comment further argues, based on the analysis that the authors chose to adopt, that reaction rate (initial $20 \mathrm{~min}$ ) differs when calculated from azide signal or from product signal, but their conclusion depends on technical details of the integration limits that they selected for quantification. Our own analysis of the data does not produce this discrepancy.

Fourth: This section repeats the argument, discussed in our introductory paragraphs, that order of applied magnetic field amplitude matters decisively. Reasonable people could perhaps disagree whether there could exist a second-order influence in principle, but our publications have explained repeatedly how we designed why the experiment to avoid this issue ${ }^{2,14}$. We reiterate that when this idea was put to experimental test, neither our data nor theirs showed a qualitative effect (Fig. 1).

Fifth: The authors disagree with concerns that had troubled prominent early users of this NMR technique ${ }^{19-20}$. We are glad that authors of this Comment consider that modern technology has solved this problem fully but we don't understand why the authors argue about a remark that we labeled as speculative. We originally proposed this speculation in the spirit of brainstorming why claims made in the authors' first comment disagree with our measurements ${ }^{1-2}$. Having now inspected the authors' raw data and learned that their data in fact agree broadly with ours, but not with their claims (Fig. 1), we recognize that this was a red herring. 
The authors would have been happier if we had used a nonlinear fit to the Stejskal-

Tanner equation. This is not standard in the literature ${ }^{19-21}$. We do not understand why the authors consider it advantageous to introduce additional parameters. This seems to be yet another matter on which reasonable people can disagree.

In conclusion, the additional factual evidence and reasoning in this Reply provide additional support to this laboratory's conclusions regarding boosted diffusion during common chemical reactions.

\section{ACKNOWLEDGMENT}

This study was supported by taxpayers of South Korea through the Institute for Basic Science (project code IBS-R020-D1). 

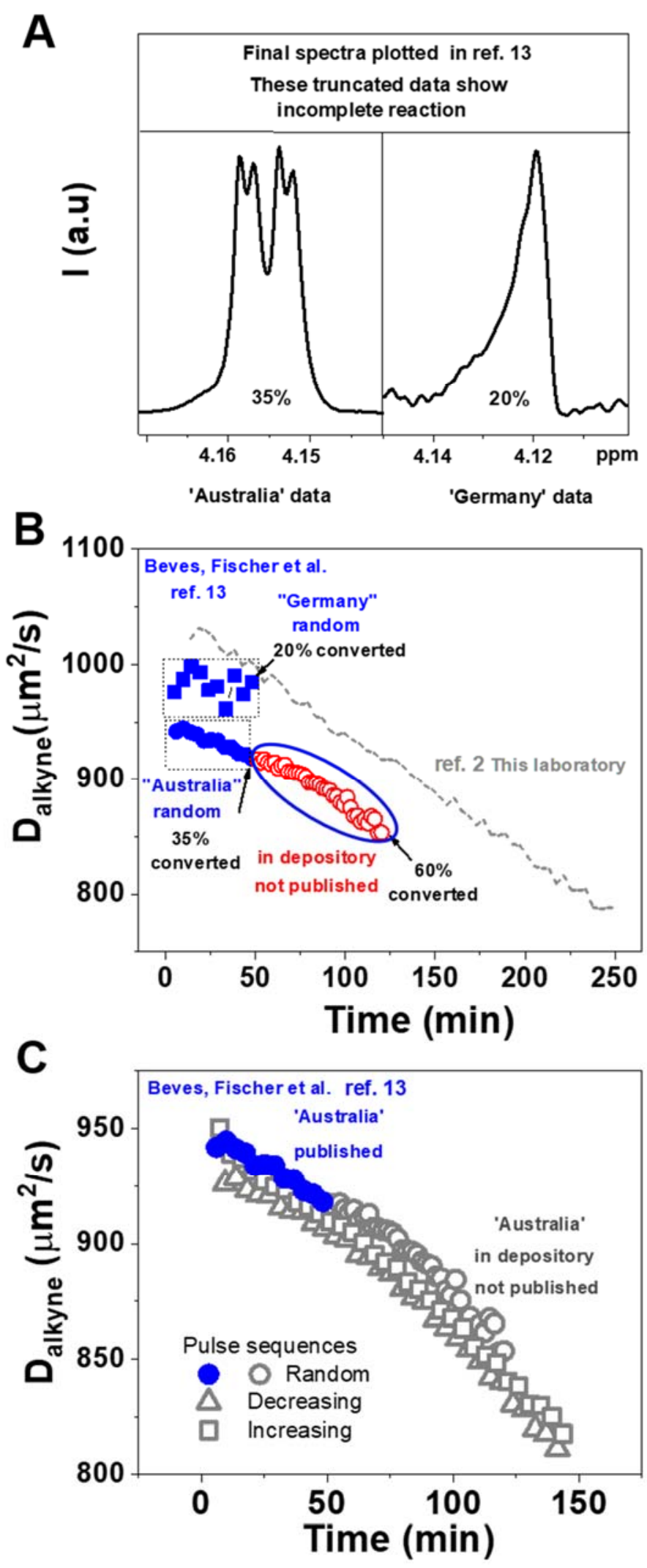
Figure 1. An earlier publication by these authors ${ }^{13}$ truncated the data. (A) Raw data the authors deposited online regarding the longest time for which they published (50 min). Inspection reveals this time to be at only $35 \%$ reaction ("Australia" data) and 20\% reaction ("Germany" data) . Note the distorted NMR peak in the "Germany" data. (B) Time-dependent diffusion coefficient of the akyne reagent, evaluated from the "Germany" (squares) and "Australia" D (circles). Note that the published data (filled symbols) omitted data the authors deposited online (open symbols). The dotted grey line shows consistency of the unpublished data with data from this laboratory $^{2}$. (C) Dependence on the sequence of gradient field application, evaluated from the "Australia" data, for the time-dependent diffusion coefficient of the alkyne reagent. The trend of time dependence is the same for random ("shuffled") (circles), increasing (squares), and decreasing (triangles) magnetic field gradients, consistent with this laboratory's finding. 


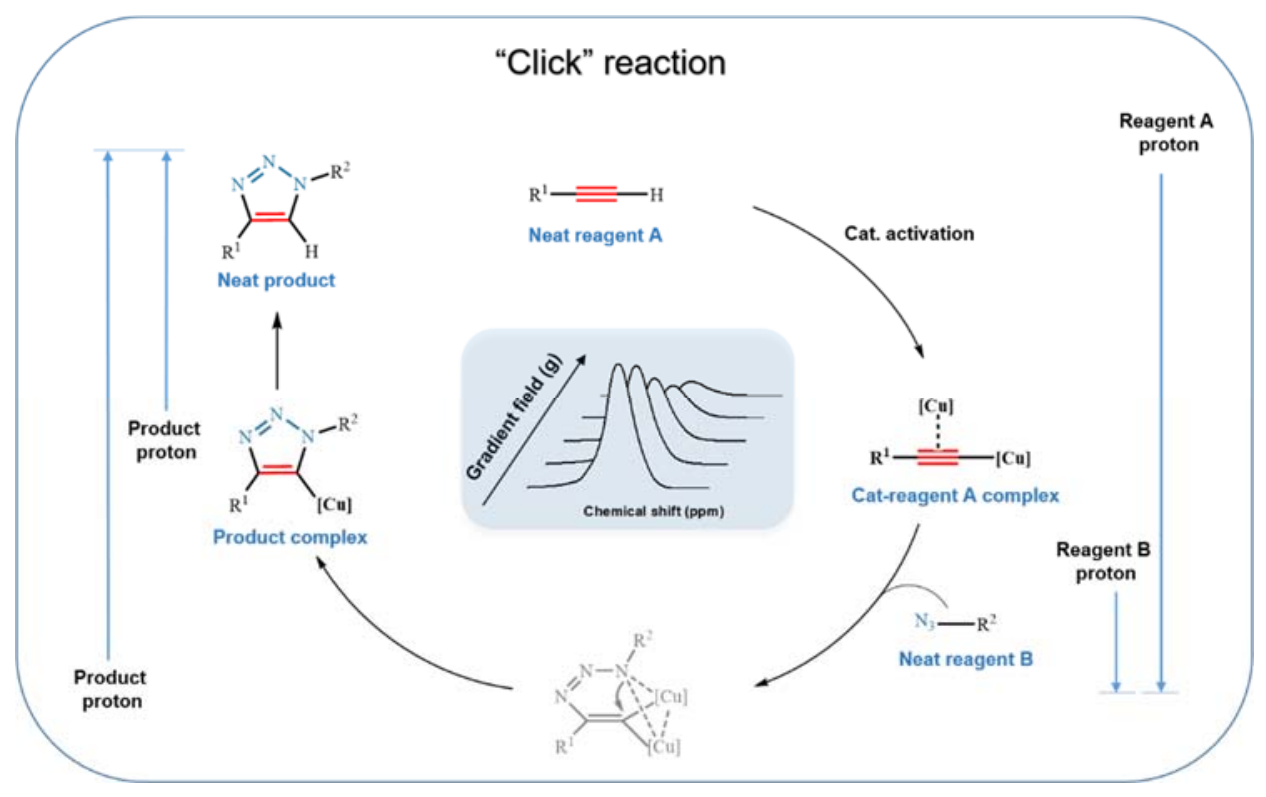

Figure 2. Some of the many reaction intermediates in the CuAAC (copper-catalyzed azidealkyne cycloaddition) click reaction. Raw data are measured using proton pulsed field-gradient NMR, shown schematically at the center of this figure. Authors of the Comment are concerned to observe that the absolute diffusion coefficient of the alkyne protons decreases below their values for pristine alkyne and no chemical reaction, but this is because they become integrated into known intermediate alkyne-copper complexes. 

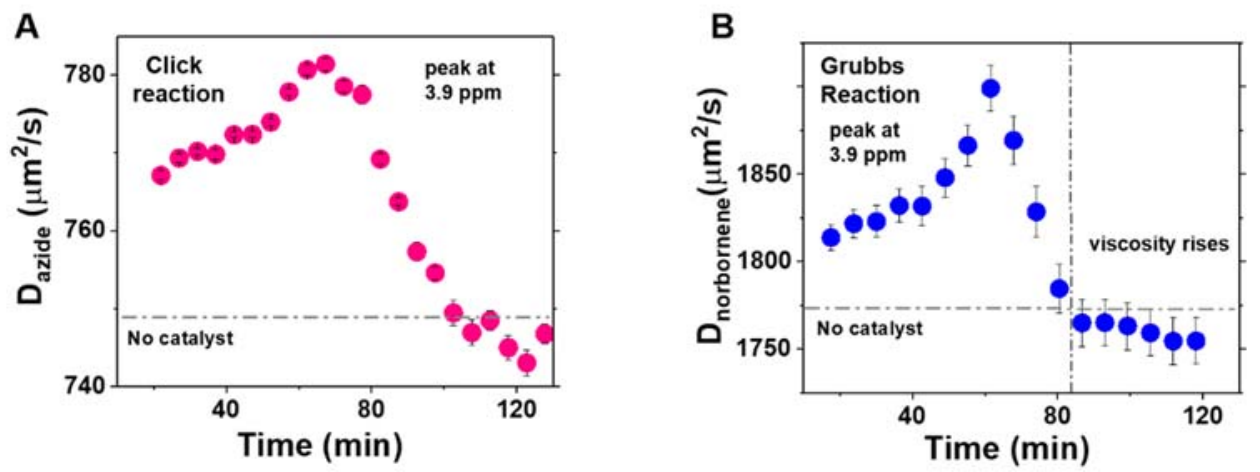

Figure 3. Examples of boosted diffusion during chemical reaction for both the click $(\mathrm{A})$ and Grubbs (B) chemical reactions when one normalizes to reagents in the absence of copper catalyst. (A) Referring to the azide peak at $3.9 \mathrm{ppm}$, the starting reaction mixture contains 250 $\mathrm{mM}$ alkyne $+300 \mathrm{mM}$ azide $+600 \mu \mathrm{L} \mathrm{D} 2 \mathrm{O}+100 \mathrm{mM}$ ascorbate $+25 \mathrm{mM} \mathrm{CuSO}_{4}$. The solution was filtered by $0.20 \mu \mathrm{m}$ PTFE filter to remove $\mathrm{Cu}$ particles produced by disproportionated reaction. Experimental condition: $600 \mathrm{MHz}$ FT-NMR (Agilent), pulse width $=11.5 \mu \mathrm{s}$, relaxation delay time $=15 \mathrm{~s}$, gradient length $=2.0 \mathrm{~ms}$, diffusion time $=50 \mathrm{~ms}$, increasing pulse sequence with convection suppression. (B) Referring to the reactant peak at $5.9 \mathrm{ppm}$, this data was deposited online to support our original publication but not previously presented graphically. ${ }^{1}$. 


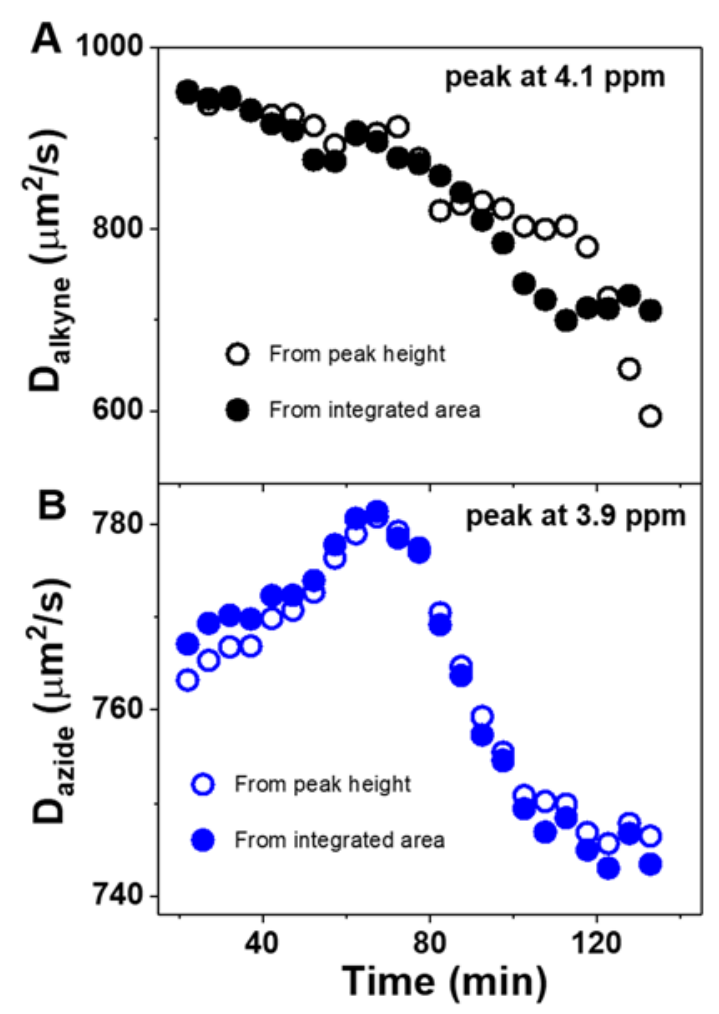

Figure 4. Technical issues do not explain away our analysis of the alkyne NMR data. Diffusion coefficient plotted against reaction time for the alkyne peak at $4.1 \mathrm{ppm}(\mathbf{A})$ and the azide peak at $3.9 \mathrm{ppm}(\mathbf{B})$ agree regardless of whether one analyzes peak height or integrated area.

Experimental condition: $600 \mathrm{MHz}$ FT-NMR (Agilent), pulse width $=11.5 \mu \mathrm{s}$, relaxation delay time $=15 \mathrm{~s}$, gradient length $=2.0 \mathrm{~ms}$, diffusion time $=50 \mathrm{~ms}$, increasing pulse sequence with convection suppression. 


\section{REFERENCES}

1. Wang, H.; Park, M.; Dong, R.; Kim, J.; Cho, Y.-K.; Tlusty, T.; Granick, S., Boosted molecular mobility during common chemical reactions. Science 2020, 369 (6503), 537.

2. Wang, H.; Huang, T.; Granick, S., Using NMR to Test Molecular Mobility during a Chemical Reaction. The Journal of Physical Chemistry Letters 2021, 12 (9), 2370-2375.

3. Muddana, H. S.; Sengupta, S.; Mallouk, T. E.; Sen, A.; Butler, P. J., Substrate Catalysis Enhances SingleEnzyme Diffusion. Journal of the American Chemical Society 2010, 132 (7), 2110-2111.

4. $\quad$ Sengupta, S.; Dey, K. K.; Muddana, H. S.; Tabouillot, T.; Ibele, M. E.; Butler, P. J.; Sen, A., Enzyme Molecules as Nanomotors. Journal of the American Chemical Society 2013, 135 (4), 1406-1414.

5. Dey, K. K.; Zhao, X.; Tansi, B. M.; Méndez-Ortiz, W. J.; Córdova-Figueroa, U. M.; Golestanian, R.; Sen, A., Micromotors Powered by Enzyme Catalysis. Nano Letters 2015, 15 (12), 8311-8315.

6. Jee, A.-Y.; Cho, Y.-K.; Granick, S.; Tlusty, T., Catalytic enzymes are active matter. Proceedings of the National Academy of Sciences 2018, 201814180.

7. Jee, A.-Y.; Dutta, S.; Cho, Y.-K.; Tlusty, T.; Granick, S., Enzyme leaps fuel antichemotaxis. Proceedings of the National Academy of Sciences 2018, 115 (1), 14.

8. Jee, A.-Y.; Tlusty, T.; Granick, S., Master curve of boosted diffusion for 10 catalytic enzymes. Proceedings of the National Academy of Sciences 2020, 117 (47), 29435.

9. $\quad$ García-López, V.; Chen, F.; Nilewski, L. G.; Duret, G.; Aliyan, A.; Kolomeisky, A. B.; Robinson, J. T.; Wang, G.; Pal, R.; Tour, J. M., Molecular machines open cell membranes. Nature 2017, 548 (7669), 567-572.

10. Lucy, F.; Jan-Philipp, G.; Günter, M.; William S., P.; Peer, F.; Jonathon, B., Errors in the Use of NMR to Test Molecular Mobility during a Chemical Reaction. The Journal of Physical Chemistry Letters 2021, in press.

11. Wang, H.; Huang, T.; Granick, S. Use or misuse of NMR to test molecular mobility during chemical reactions. https://zenodo.org/record/4515126.

12. Wang, H.; Park, M.; Dong, R.; Kim, J.; Cho, Y.-K.; Tlusty, T.; Granick, S. Raw data for Wang et al., "Boosted molecular mobility during common chemical reactions," Science (2020) 369, 537-541.

https://zenodo.org/record/4553903.

13. Günther, J.-P.; Fillbrook, L. L.; MacDonald, T. S. C.; Majer, G.; Price, W. S.; Fischer, P.; Beves, J. E., Comment on "Boosted molecular mobility during common chemical reactions". Science 2021, 371 (6526), eabe8322.

14. Wang, H.; Park, M.; Dong, R.; Kim, J.; Cho, Y.-K.; Tlusty, T.; Granick, S., Response to Comment on "Boosted molecular mobility during common chemical reactions". Science 2021, 371 (6526), eabe8678.

15. Günther, J.-P. F.; Fillbrook, L. L.; MacDonald, T. S. C.; Majer, G.; Price, W. S.; Fischer, P.; Beves, J. E. Raw data for: Comment on "Boosted molecular mobility during common chemical reactions".

https://zenodo.org/record/4628353.

16. Huang, T.; Li, B.; Wang, H.; Granick, S., Linking Chemical Reaction Intermediates of the Click Reaction to Their Molecular Diffusivity. ChemRxiv 2021, doi.org/10.26434/chemrxiv.14740563.v1.

17. Graf, V.; Noack, F.; Béné, G. J., Proton spin T1 relaxation dispersion in liquid H2O by slow proton exchange. The Journal of Chemical Physics 1980, 72 (2), 861-863.

18. Bloembergen, N.; Purcell, E. M.; Pound, R. V. J. P. r., Relaxation effects in nuclear magnetic resonance absorption. Physical Review 1948, 73 (7), 679.

19. Johnson Jr, C. S. J. P. i. N. M. R. S., Diffusion ordered nuclear magnetic resonance spectroscopy: principles and applications. Prog. Nucl. Magn. Reson. Spectrosc. 1999, 34 (3-4), 203-256.

20. Pagès, G.; Gilard, V.; Martino, R.; Malet-Martino, M., Pulsed-field gradient nuclear magnetic resonance measurements (PFG NMR) for diffusion ordered spectroscopy (DOSY) mapping. Analyst 2017, 142 (20), 37713796.

21. Stejskal, E. O.; Tanner, J. E., Spin Diffusion Measurements: Spin Echoes in the Presence of a Time Dependent Field Gradient. The Journal of Chemical Physics 1965, 42 (1), 288-292. 